\title{
Intrinsic Connectivity Networks, Alpha Oscillations, and Tonic Alertness: A Simultaneous Electroencephalography/ Functional Magnetic Resonance Imaging Study
}

\author{
Sepideh Sadaghiani, ${ }^{1,2,3,5}$ René Scheeringa, ${ }^{1,2,3}$ Katia Lehongre, ${ }^{4}$ Benjamin Morillon, ${ }^{4}$ Anne-Lise Giraud, ${ }^{4}$ \\ and Andreas Kleinschmidt ${ }^{1,2,3}$ \\ ${ }^{1}$ INSERM Unité 992, Cognitive Neuroimaging, F-91191 Gif-sur-Yvette, France, ${ }^{2}$ Commissariat à l'Energie Atomique, Direction des Sciences du Vivant, \\ Institut d'Imagerie Biomédicale, NeuroSpin, F-91191 Gif-sur-Yvette, France, ${ }^{3}$ Université Paris-Sud, F-91405 Orsay, France, ${ }^{4}$ INSERM Unité 960 , \\ Laboratoire de Neurosciences Cognitives, Ecole Normale Supérieure, F-75005 Paris, France, and 5International Max Planck Research School of Neural and \\ Behavioural Sciences, University of Tübingen, D-72074 Tübingen, Germany
}

Trial-by-trial variability in perceptual performance on identical stimuli has been related to spontaneous fluctuations in ongoing activity of intrinsic functional connectivity networks (ICNs). In a paradigm requiring sustained vigilance for instance, we previously observed that higher prestimulus activity in a cingulo-insular-thalamic network facilitated subsequent perception. Here, we test our proposed interpretation that this network underpins maintenance of tonic alertness. We used simultaneous acquisition of functional magnetic resonance imaging ( $\mathrm{FMRI}$ ) and electroencephalography (EEG) in the absence of any paradigm to test an ensuing hypothesis, namely that spontaneous fluctuations in this ICN's activity (as measured by fMRI) should show a positive correlation with the electrical signatures of tonic alertness (as recorded by concurrent EEG). We found in human subjects ( 19 male, 7 female) that activity in a network comprising dorsal anterior cingulate cortex, anterior insula, anterior prefrontal cortex and thalamus is positively correlated with global field power (GFP) of upper alpha band (10-12 Hz) oscillations, the most consistent electrical index of tonic alertness. Conversely, and in line with earlier findings, alpha band power was negatively correlated with activity in another ICN, the so-called dorsal attention network which is most prominently involved in selective spatial attention. We propose that the cingulo-insular-thalamic network serves maintaining tonic alertness through generalized expression of cortical alpha oscillations. Attention is mediated by activity in other systems, e.g., the dorsal attention network for space, selectively disrupts alertness-related suppression and hence manifests as local attenuation of alpha activity.

\section{Introduction}

Recent functional neuroimaging studies have shown that evoked response variability correlates with ongoing activity fluctuations and that this variability transpires into perceptual variability (for review, see Sadaghiani et al., 2010). As a function of the paradigm, effects of ongoing activity on perceptual performance have been observed both locally in accordingly specialized areas (Hesselmann et al., 2008a,b; Sadaghiani et al., 2009) and in distributed spatial patterns that resemble resting-state or intrinsic connectivity networks (ICNs) (Boly et al., 2007; Sadaghiani et al., 2009). In some ICNs higher prestimulus activity facilitates and in others it deteriorates perceptual performance on upcoming stimuli. In a recent study using near-threshold acoustic stimuli (Sadaghiani et al., 2009), we found that prestimulus signal in frontoparietal regions including the intraparietal sulcus (IPS) and frontal eye

Received Feb. 18, 2010; revised May 19, 2010; accepted June 21, 2010.

This work was funded by the Agence Nationale de le Recherche, France (ANR Grant SPONTACT). S.S. is supported by the Friedrich-Ebert Foundation (Germany). Data were acquired at the Centre de Neuroimagerie de Recherche (CENIR), Hôpital Pitié-Salpêtrière, Paris, France.

Correspondence should be addressed to Sepideh Sadaghiani, INSERM Unité 992, NeuroSpin, CEA/SAC/DSV/L2BM, Bât 145, Point Courrier 156, F-91191 Gif-sur-Yvette, France. E-mail: sepideh.sadaghiani@gmail.com.

DOI:10.1523/JNEUROSCI.1004-10.2010

Copyright $\odot 2010$ the authors $\quad 0270-6474 / 10 / 3010243-08 \$ 15.00 / 0$ fields biased toward missing subsequent stimuli whereas signal in auditory cortex but also in a network comprising dorsal anterior cingulate cortex (dACC), anterior insula and thalamus facilitated their detection. The former network is well characterized but the latter has remained more elusive both in terms of topography and function. Several studies have defined largely similar networks with slightly varying numbers and locations of constituent elements (Vincent et al., 2008). And others have attempted to dissect it into subnetworks linked to executive control, task-set maintenance, or salience (Dosenbach et al., 2006, 2007; Seeley et al., 2007). Yet, our paradigm with simple detection of meaning-free sparse stimuli only required maintaining tonic alertness. In accord with this interpretation, several studies that explicitly probed tonic alertness found effects in similar brain structures as we did (Sturm and Willmes, 2001; Sturm et al., 2004).

Here, we sought to further corroborate this functional interpretation. We based our investigation on results from related settings used to characterize the electroencephalographic signatures of tonic alertness in ongoing activity (Gath et al., 1983; Makeig and Inlow, 1993; Haig and Gordon, 1998). We reasoned that if our functional interpretation was correct, in the absence of any paradigm fluctuations in vigilance as indicated in the electroencephalogram should be associated with fluctuations in activity 
of this putative alertness network and vice versa. To test this hypothesis, we analyzed findings from simultaneous EEG and fMRI recordings during prolonged task-free resting state sessions. Previous studies have established correlations between ongoing activity in resting state networks and the expression of oscillatory EEG activity in different frequency bands (Laufs et al., 2003, 2006; Scheeringa et al., 2008). Remarkably, however, not even the most comprehensive attempt so far (Mantini et al., 2007) has reported findings for the network that we postulate to be involved in maintaining tonic alertness. Here, we fill this gap by showing that activity in a cingulo-insular-thalamic network is positively correlated with the GFP of oscillations in the alpha (and to some extent beta) band of the simultaneously recorded EEG. This finding matches EEG signatures of tonic alertness (Gath et al., 1983; Makeig and Inlow, 1993; Makeig and Jung, 1995; Haig and Gordon, 1998; Lockley et al., 2006; Dockree et al., 2007) and hence corroborates our previous functional interpretation of at least one functional role of this brain network.

\section{Materials and Methods}

Subjects and data acquisition. Twenty-seven healthy volunteers (ages: 1929; 8 females; 5 left-handed; written informed consent) underwent simultaneous EEG and blood oxygen level-dependent (BOLD) fMRI. Data from one subject (female, right-handed) were excluded from analysis due to strong head motion. Subjects were requested to rest with eyes closed, stay awake, and avoid movement.

Three 10 min sessions yielded 300 echoplanar fMRI image volumes each (Tim-Trio; Siemens, 40 transverse slices, voxel size $=3 \times 3 \times 3$ $\mathrm{mm}^{3}$; repetition time $=2000 \mathrm{~ms}$; echo time $=50 \mathrm{~ms}$, field of view $\left.=192\right)$ and continuous EEG data recorded at $5 \mathrm{kHz}$ from 62 scalp sites (Easycap electrode cap) using MR-compatible amplifiers (BrainAmp MR and Brain Vision Recorder software; Brain Products). Two additional electrodes (EOG and ECG) were placed under the right eye and on the collarbone. Impedances were kept under $10 \mathrm{k} \Omega$ and EEG was time-locked with the scanner clock. A 7 min anatomical T1-weighted magnetization-prepared rapid acquisition gradient echo sequence ( 176 slices, field of view $=256$, voxel size $=$ $1 \times 1 \times 1 \mathrm{~mm}$ ) was acquired at the end of scanning.

fMRI preprocessing. We used statistical parametric mapping (SPM5, Wellcome Department of Imaging Neuroscience, London, UK; www.fil. ion.ucl.ac.uk) for image preprocessing (realignment, coregistration with the structural image, segmentation and normalization to Montreal Neurological Institute stereotactic space and reslicing to $2 \times 2 \times 2 \mathrm{~mm}$, spatial smoothing with a $6 \mathrm{~mm}$ full-width half-maximum isotropic Gaussian kernel for single subject as well as group analyses) and estimation of general linear models (GLMs).

fMRI intrinsic functional connectivity. For calculation of ICNs seed regions were defined as the (subject-specific) gray-matter voxels in a sphere of $10 \mathrm{~mm}$ radius around peaks extracted from previous studies; right intraparietal sulcus [ $27-5849$ ] for the dorsal attention system (Fox et al., 2006) and dACC [ $\left.\begin{array}{lll}0 & 15 & 40\end{array}\right]$ for the intrinsic alertness system [centered between [ 61542$]$ and [ -61539$]$ as found in intrinsic connectivity analysis by Sadaghiani et al. (2009)]. For each seed region the high-pass filtered $(1 / 128 \mathrm{~Hz})$ signal time course was averaged across all respective voxels and used as a regressor of interest in a separate GLM. Nuisance covariates of no interest included the global signal of three separate brain compartments (all white-matter voxels, all gray-matter voxels and all CSF voxels), all out-of-brain voxels as well as 6 head-motion parameters. Contrast images corresponding to the seed regressor were created for each subject and entered into a second level one-sample $t$ test. Maps were rendered onto an inflated canonical average brain (FreeSurfer, CorTech, http://surfer.nmr.mgh.harvard.edu).

For the tonic alertness and dorsal attention ICNs as well as their individual constituent regions, BOLD time courses were extracted (group-level contrast, $p<0.01$ family-wise error (FWE)-corrected, extent $>150$ voxels) for regression analysis with spectrally resolved EEG power time courses (see below). Insula, thalamus and basal ganglia constituted a large cluster and were thus separated by masking with corresponding anatomical masks generated by PickAtlas (Wake Forest University School of Medicine, http://www.fmri.wfubmc.edu/cms/software). Cortical regions were further restricted to the subject-specific intrinsic connectivity maps (first-level, $p<0.005$ uncorrected, extent $>50$ voxels) and gray-matter mask.

EEG preprocessing. We used EEGlab v.7 (http://sccn.ucsd.edu/eeglab) and the FMRIB plug-in (http://users.fmrib.ox.ac.uk/ rami/fmribplugin) for gradient and pulse artifact subtraction. In two subjects, one of three rest sessions each was excluded due to insufficient EEG quality. The data were subsequently downsampled to $250 \mathrm{~Hz}$ and re-referenced to a common average reference. The original reference electrode was recalculated as $\mathrm{FCz}$, yielding a total of $63 \mathrm{EEG}$ channels.

Network-based correlation analysis with the EEG power spectrum. Timefrequency analysis was computed in Fieldtrip (Donders Institute for Brain Cognition and Behavior; http://www.ru.nl/fcdonders/fieldtrip). The time-frequency analysis of power for the regression of regional fMRI signal onto EEG was performed for all the frequencies up to $30 \mathrm{~Hz}$ in steps of $0.5 \mathrm{~Hz}$. For this we used a multitaper approach (Mitra and Pesaran, 1999) integrating the power between $1 \mathrm{~Hz}$ below and $1 \mathrm{~Hz}$ above the center frequency, and over a $2 \mathrm{~s}$ time-interval. This estimate was repeated for every $0.4 \mathrm{~s}$. The power time courses were converted to $z$-scores. As artifact rejection strategy, $z$-scores larger than 4 were removed from the time-series. The remaining values were again $z$-transformed.

For each channel-frequency combination a separate design matrix was constructed, that included the hemodynamic response function (HRF)convolved power time course for that specific channel-frequency combination. We included signal of three separate brain compartments (all white-matter voxels, all gray-matter voxels and all CSF voxels), of all out-of-brain voxels and of the motion parameters as nuisance covariates. This yielded for each frequency channel point a beta value for the EEG power regressor, which was tested at group level using a single sample $t$ test against zero. This analysis was repeated for the fMRI time course of the tonic alertness and dorsal attention ICNs as well as each constituent region.

For the signals extracted from the entire ICNs, multiple-comparison correction was performed using a cluster-based randomization procedure (Maris and Oostenveld, 2007). This effectively controls the Type-1 error rate in a situation involving multiple comparisons. The procedure allows for the use of user defined test statistics tailored to the effect of interest within the framework of a cluster-based randomization test. Here, we used a single sample $t$ test against zero, giving uncorrected $p$-values. All data points that did not exceed the preset significance level of $p<0.05$ were zeroed. Clusters of adjacent non-zero channelfrequency points were computed, and for each cluster a cluster-level test statistic was calculated by taking the sum of all the individual $t$-statistics within that cluster. This statistic was entered in the cluster-based randomization procedure. For each randomization the lowest negative and highest positive cluster-sum entered a reference distribution. Clusters that fell within the extreme $2.5 \%$ of the negative and positive randomization distribution were labeled as significant. In this case, 1000 randomizations were used to compute the reference distribution. Channels separated by $<4 \mathrm{~cm}$ were labeled as being adjacent channels. Channel positions were recorded for each subject. From these data the average distances over subjects between channel pairs were computed.

Whole-brain fMRI analysis using band-limited EEG-regressors. Power estimation for the fMRI whole-brain analysis probing BOLD signal correlation with band-specific EEG power fluctuations was based on a Hanning tapered FFT on $2 \mathrm{~s}$ windows, resulting in a $0.5 \mathrm{~Hz}$ resolution in the power spectrum. Power for each time-point was subsequently averaged over all channels. Upper alpha band was defined using the physiological criterion of individual alpha peak frequency as an anchor and $2 \mathrm{~Hz}$ width as suggested by Klimesch (1999). The peak was determined as the peak power value between 8 and $12 \mathrm{~Hz}$ after removing a 1/frequency trend from the spectrum. The global power time course of upper alpha (from individual peak bin included to $1.5 \mathrm{~Hz}$ above peak) and beta $(15-25 \mathrm{~Hz}$ excluding the main residual gradient artifact frequency of $20 \mathrm{~Hz} \pm 0.5$ $\mathrm{Hz}$ ) were used for regression analyses.

The power time courses were converted to $z$-scores. As artifact rejection strategy, $z$-scores larger than 4 were removed from the time-series. The remaining values were again $z$-transformed. 

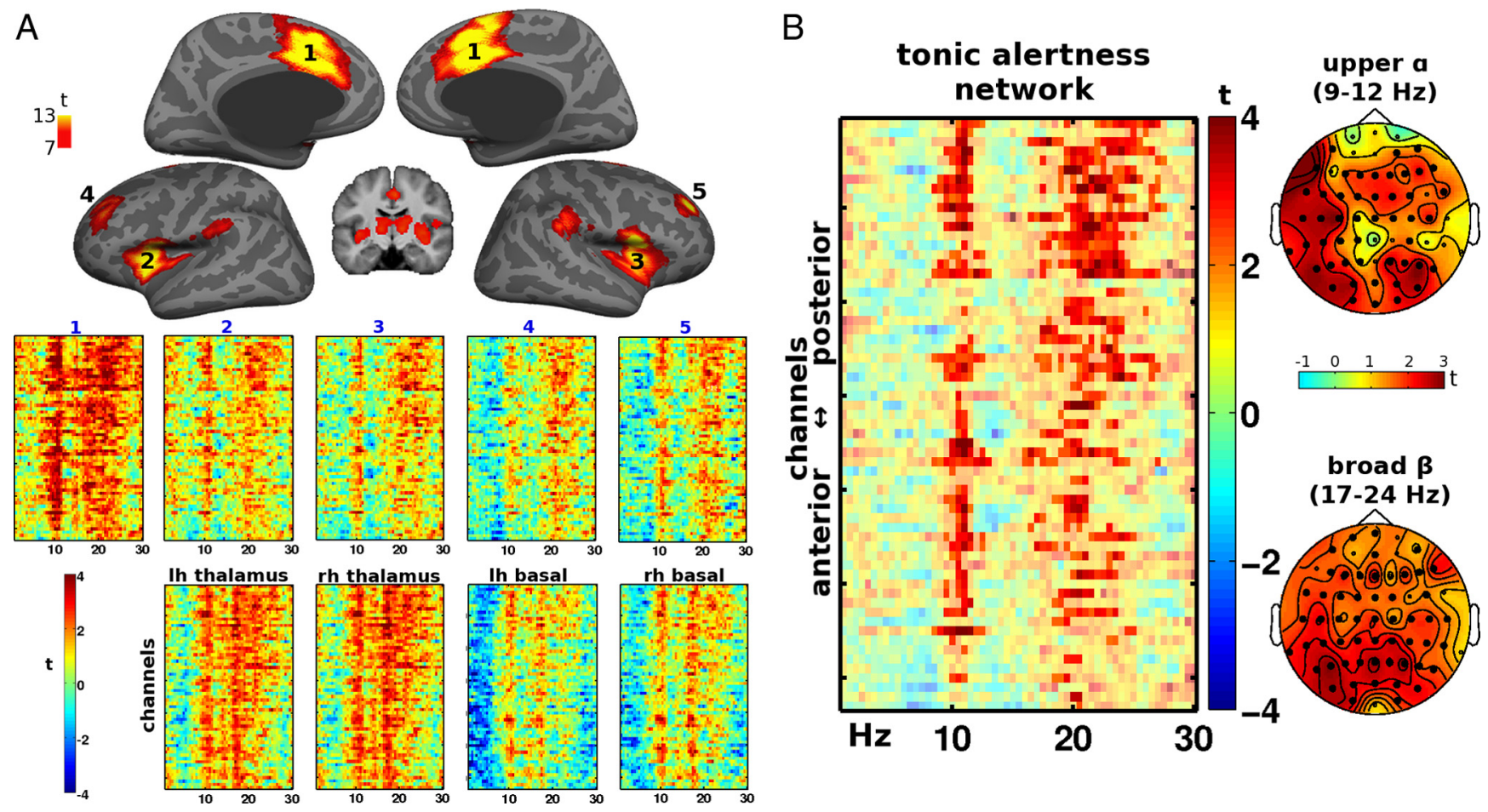

Figure 1. Channel-frequency spectra for the tonic alertness network. The tonic alertness ICN was defined by applying a seed in $\mathrm{dACC}(\boldsymbol{A})$. fMRI signal time courses extracted from this network were used for regression analyses with EEG power fluctuations across the spectrum of $1-30 \mathrm{~Hz}$. For all spectra, the $x$-axis represents EEG frequency, and EEG-channels are organized on the $y$-axis from top to bottom in a posterior-to-anterior order. Consistent across all individual regions $(\boldsymbol{A})$ and for the entire network $(\boldsymbol{B})$, positive correlations arise rather selectively in the high alpha band and more broadly in the beta band. Effects occur rather globally across the majority of EEG-channels and are furthermore characterized by distributed topographies for the network $(\boldsymbol{B})$. $\boldsymbol{A}$, The ICN is defined at $p<0.01$ FWE-corrected, extent $>150$ voxels, mapped on a canonical average inflated cortical surface and a coronal section $[y=-12]$. The individual cortical regions' spectra are numbered as depicted on the rendered ICN. Spectra for subcortical areas are titled respectively. $\boldsymbol{B}$, The translucent mask represents significance after correction. Bold closed channels in the topographies are part of the significant cluster.

The GFP time courses of upper alpha and beta bands were entered each into a separate GLM as a parametrically modulated regressor with stick functions modeling the event duration. Hereby, the power time courses were convolved with the canonical HRF function. Again, signal of three separate brain compartments (all white-matter voxels, all gray-matter voxels and all CSF voxels), of all out-of-brain voxels and of the motion parameters served as nuisance covariates. Contrast images corresponding to the parametric regressor were created for each subject and entered into a second level one-sample $t$ test. An auxiliary uncorrected threshold of $p<0.005$ and extent $>100$ voxels was used. Exceptions where a higher threshold was used for more fine-grained analysis are stated explicitly. Cluster-level corrected results are reported at $p<0.05$ corrected threshold.

\section{Results}

To define the intrinsic connectivity network putatively related to tonic alertness we applied a seed region in the dACC. As in earlier studies (Dosenbach et al., 2007; Seeley et al., 2007; Sadaghiani et al., 2009) this resting state functional connectivity network contained bilateral insula, dACC, anterior prefrontal cortex (aPFC), thalamus and basal ganglia. We extracted the fMRI signal time course of this network as well as of its individual constituent regions and performed regression onto EEG power fluctuations across the spectrum of $1-30 \mathrm{~Hz}$ (Fig. 1). Positive correlations with the BOLD time course occurred rather selectively in the high alpha band $(\sim 10 \mathrm{~Hz}$ and above; cf. individual alpha peak frequency $\approx 10 \mathrm{~Hz})$ and more broadly in the beta band $(\sim 17-24$ $\mathrm{Hz}$ ). This effect was expressed in the spectral patterns of all constituent regions (Fig. 1A) and was significant in the network (Fig. $1 B$ ). Conversely, these correlations did not appear to be constrained to subsets of electrodes but occurred rather globally across the majority of EEG-channels. This global character was further confirmed in distributed effects on topography maps (Fig. $1 B$ ). The channel-frequency spectra for the network are presented in supplemental Figure 1 (available at www.jneurosci. org as supplemental material) for all 26 individual subjects. The spectral distribution of correlation of ICN activity with EEG oscillations is in accord with previously described EEG signatures of alertness in the alpha and beta range (Townsend and Johnson, 1979; Gath et al., 1983; Belyavin and Wright, 1987; Makeig and Inlow, 1993; Makeig and Jung, 1995; Haig and Gordon, 1998; Lockley et al., 2006).

For comparison, we next mapped in our data set a different ICN, the dorsal attention system, by applying a seed in right IPS and confirmed it to contain bilateral IPS, frontal eye fields and middle temporal motion-sensitive cortex (MT+) (Fig. 2A). Again, the equivalent analysis was conducted by performing regression analyses with the spectral EEG data and the BOLD time course of the dorsal attention network and its individual constituent regions. This analysis revealed a selective negative correlation in the alpha and beta bands in all constituent regions (Fig. $2 A$ ) as well as the network (Fig. $2 B$ ). The negative correlations occurred in a broader frequency range and with a lower center frequency $(\sim 7-10 \mathrm{~Hz})$ compared with the positive correlations of high alpha observed in the intrinsic alertness network. The negative beta correlations occurred in a more confined and lower frequency band $(\sim 15-18 \mathrm{~Hz})$ compared with the broad positive beta correlations of the tonic alertness network.

To address the topographic specificity of our network-based correlations with the EEG spectrum, we next used band-specific 

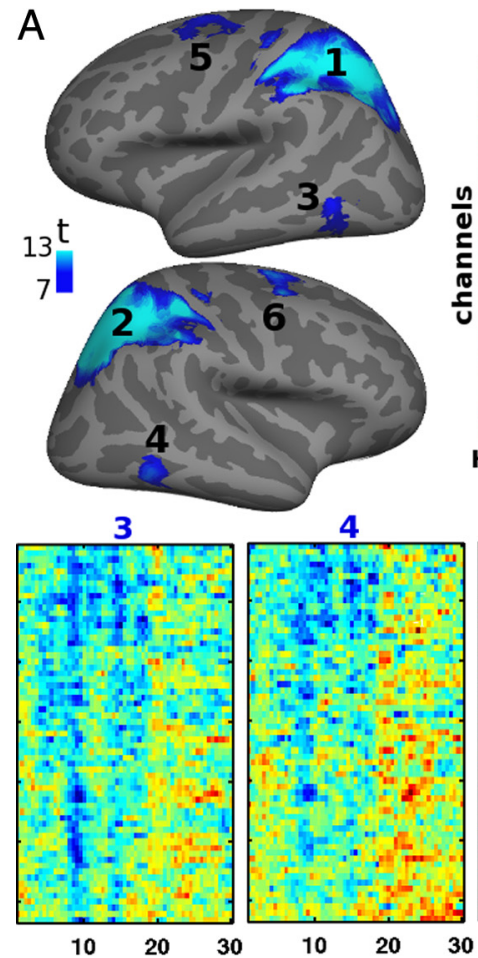
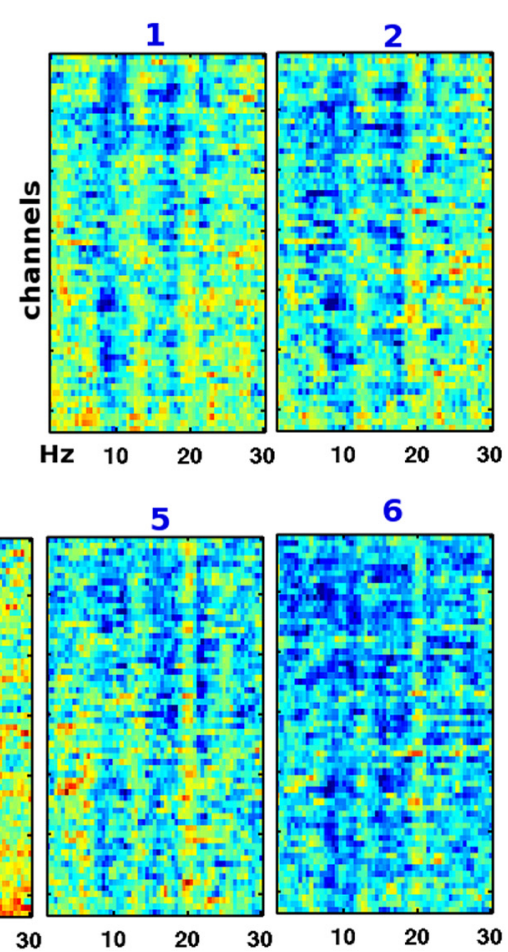

B

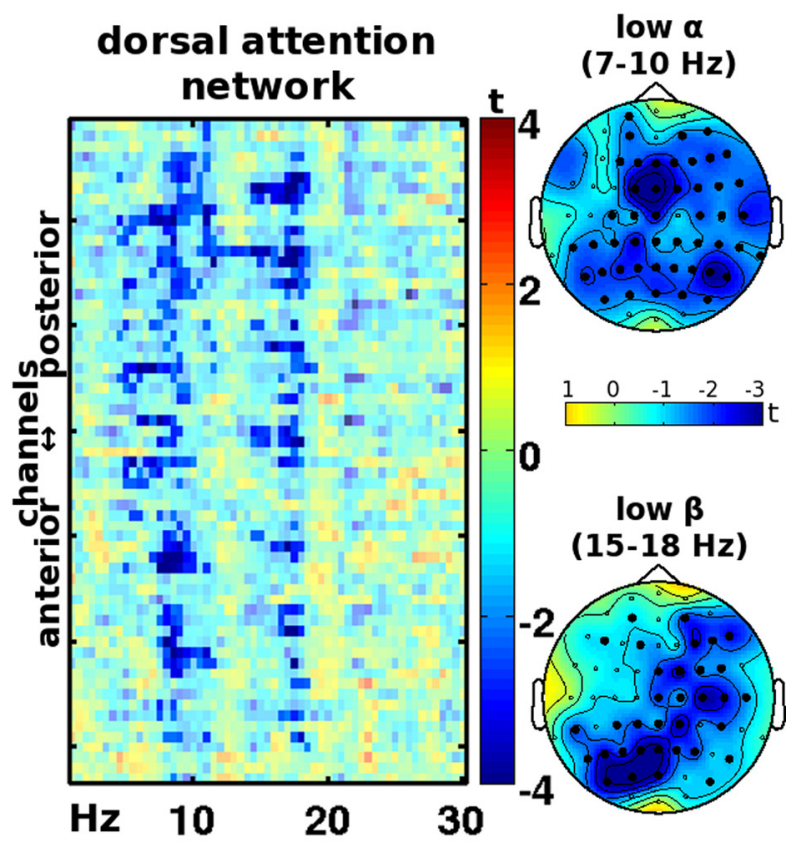

Figure 2. Channel-frequency spectra for the dorsal attention network. Dorsal attention ICN was defined by applying a seed in right IPS (A). fMRI signal time courses extracted from this network were used for regression analyses with EEG power fluctuations across the spectrum of $1-30 \mathrm{~Hz}$. Correlations arise exclusively negatively in lower alpha and beta bands consistently across all regions of the network $(\boldsymbol{A})$ and for BOLD signal averaged across the entire network $(\boldsymbol{B})$. Thresholding and visualization equivalent to Figure 1.

EEG regressors to map correlations with local fMRI signal across the whole-brain volume. The previously described spectral correlation with the tonic alertness system highlighted an effect in the so-called upper alpha band, a range that has also been linked to cognitive control and performance in previous EEG studies (Klimesch, 1999; Klimesch et al., 2007). We targeted for EEGcorrelated fMRI analyses an upper $2 \mathrm{~Hz}$ band of alpha frequency with a lowerbound anchor on individually determined peak frequency (on average $9.77 \mathrm{~Hz} \pm 0.74$ ). We tested where in the brain GFP fluctuations in the upper alpha frequency correlate positively with neural activity fluctuations as measured by BOLD signal.

The results of the whole-brain correlation with GFP of upper alpha are shown in Figure $3 A$. Positive correlations with the BOLD signal displayed a spatial pattern that strongly overlaps with the separately defined tonic alertness network (cf. Fig. $1 A$ ). These effects reached significance (cluster-level corrected, auxiliary uncorrected $p<0.001$ ) in dACC ([2, 22, 40], $p<0.001, z$-score 3.96, 801 voxels), a subcortical cluster bilaterally including thalamus and caudate nucleus ( $p<0.001, z$-score up to $4.69,1531$ voxels; caudate: [6, 24, 4], [-20 - 22 24]; thalamus: [ $-2-812]$, [2 - 14 12]), right anterior insula $([42,8,6], p=0.003, z$-score $4.36,243$ voxels $)$ and right aPFC ([34, 42, 22], $p=0.004, z$-score 4.09, 228 voxels). Further correlation with upper alpha band activity was observed in the cerebellum $([26,-38,-30], p=0.028, z$-score $4.58,150$ voxels; and $[-24,-74,-24], p<0.001, z$-score 4.37, 2096 voxels). Given previous concurrent EEG and fMRI studies in-

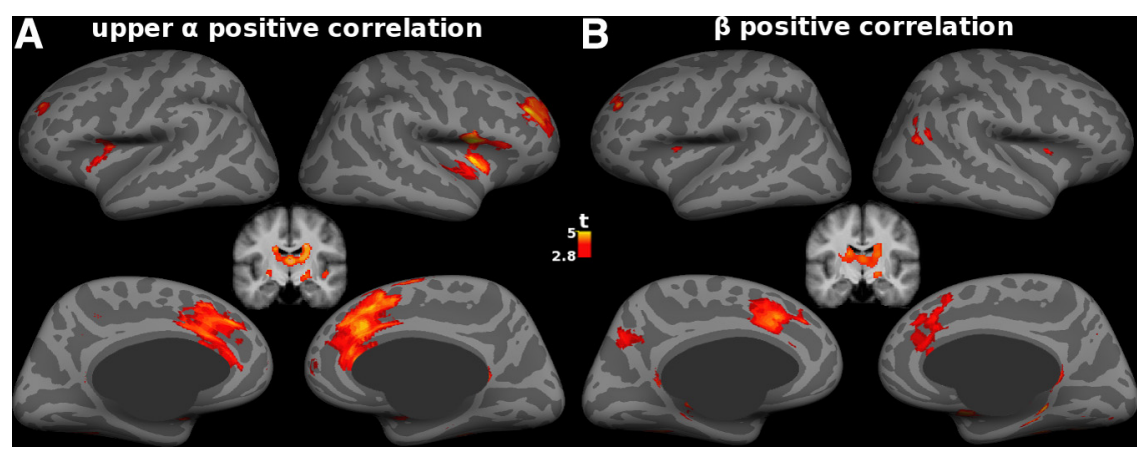

Figure 3. Positive correlation of activity with the global field power of oscillations in the alpha and beta bands. $\boldsymbol{A}$, Positive correlations with upper alpha band power arise selectively within regions of the tonic alertness network including dACC, right intrinsic alertness network, notably $\mathrm{dACC}$ and subcortical areas. $p<0.005$ uncorrected, extent $>100$ voxels, mapped on a canonical average inflated cortical surface and a coronal section $[y=-12]$.

cluding our own that have emphasized interindividual variability (Gonçalves et al., 2006; Laufs et al., 2006), we illustrate the homogeneity of the established effect across subjects in supplemental Figure 2 (available at www.jneurosci.org as supplemental material).

Given the aforementioned EEG studies also linking beta band oscillations to sustained performance (Townsend and Johnson, 1979; Belyavin and Wright, 1987) we next performed analogous analyses with EEG time courses from that frequency band. Global beta power $(15-25 \mathrm{~Hz})$ positively correlated with some areas of the intrinsic alertness network (Fig. 3B). These effects were weaker in size and extent than those observed for the correlations with upper alpha and achieved significance only in dACC ( [ -12 , $22,34], p<0.001, z$-score $3.68,804$ voxels) and a large bilateral subcortical cluster including thalamus and caudate $(p<0.001$, 


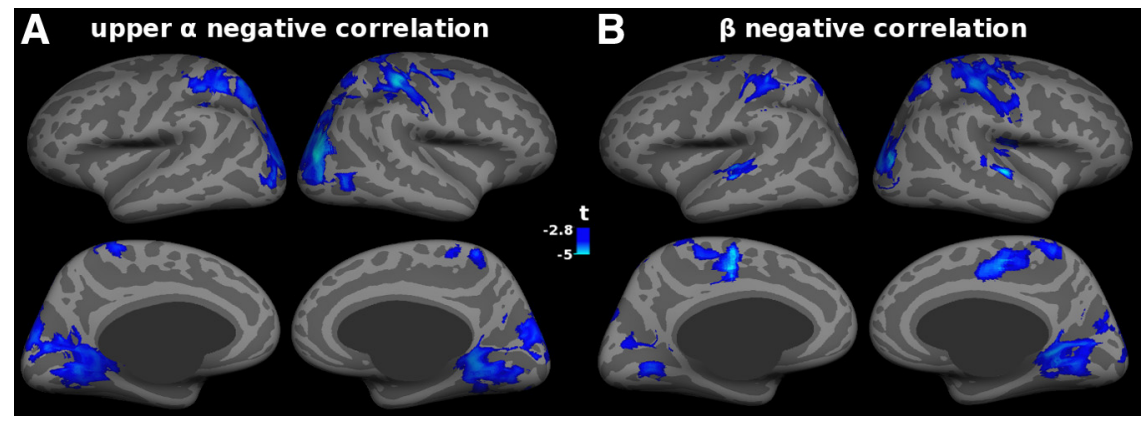

Figure 4. Negative correlation of activity with the global field power of oscillations in the alpha and beta bands. $A$, Negative correlations with upper alpha band power occur in the dorsal attention network and visual cortex, especially dorsal visual areas. $\boldsymbol{B}$, Negative correlations with beta band power are observed in right extrafoveal and dorsal visual cortex as well as sensorimotor areas. Thresholding and visualization equivalent to Figure 3.
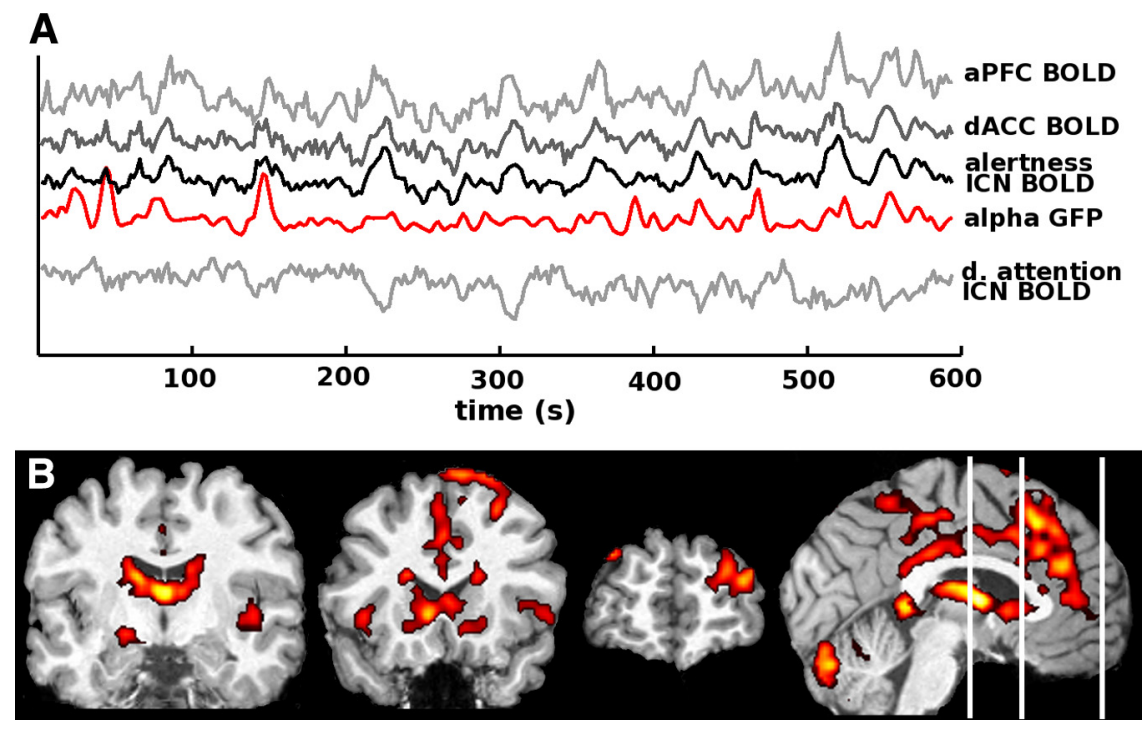

Figure 5. Signal time courses and voxelwise mapping of the correlation to upper alpha power for an individual subject. $\boldsymbol{A}$, Concurrent EEG and BOLD signal time courses for a session of $10 \mathrm{~min}$. Red: The GFP time course of the individual upper alpha band after convolution with the canonical HRF. Black/gray, Raw BOLD signal time course for the tonic alertness ICN and two of its individual regions (aPFC and $\mathrm{AACC}$ ) as well as the dorsal attention ICN as labeled. The global gray-matter BOLD signal was subtracted from these traces. The regions correspond to those visualized on the cortical surface in Figure $1 A$ and Figure $2 A$, respectively. $\boldsymbol{B}$, Voxelwise mapping of the correlation to upper alpha visualized on the subject's structural image. Positive correlations with upper alpha band power arise among others in $\mathrm{AACC}$, right and left anterior insula, right and left aPFC, thalamus and caudate. This first-level effect corresponds to the group-level analysis in Figure $3 A$. White lines on the sagittal view indicate the position of the three coronal slices ( $y=-10,16$ and 56 , respectively). $p<0.005$ uncorrected, extent $>100$ voxels.

$z$-score up to $4.05,3342$ voxels; caudate: $[-20,-46,18]$, [26 6 $12]$; thalamus $[0-1616],[-2-610])$ as well as in the cerebel$\operatorname{lum}$ ( $p<0.001, z$-score up to $4.97,7340$ voxels).

We also probed negative correlations with upper alpha band activity and obtained a spatial pattern of fMRI signal fluctuations that includes the dorsal attention system but also areas belonging to the visual system, especially dorsal areas (Fig. 4A). In accord with previous related studies (Goldman et al., 2002; Moosmann et al., 2003; Laufs et al., 2006), these effects reached significance (cluster-level corrected, auxiliary uncorrected $p<0.001$ ) in right and left dorsal visual cortex $([38,-82,16], p<0.001, z$-score $4.15,752$ voxels; and $[-30,-88,18], p=0.02, z$-score $3.54,165$ voxels), right superior parietal lobe ([46, $-28,58], p<0.001$, $z$-score 4.03, 409 voxels), left intraparietal sulcus (IPS; [ -28 , $-64,62], p=0.02, z$-score $3.67,156$ voxels) and extrastriate visual areas of bilateral lingual gyri $([22,-50,0], p=0.005$, $z$-score $3.61,218$ voxels; and $[-14,-62,6], p=0.037, z$-score
$3.44,141$ voxels). For the beta band, negative correlations were found in right, most dominantly dorsal, visual cortex ([36, $-82,14], p<0.001, z$-score 3.92, 2509 voxels), as well as in sensorimotor areas (Yuan et al., 2010) including bilateral mesial motor area $([-12,-10,48]$, $p<0.001, z$-score 4.19, 933 voxels) and right and left sensorimotor cortex ([50, -32, 58], $p<0.001, z$-score 3.71, 2500 voxels; and $[-46,-36,62], p<0.001$, $z$-score 3.76, 864 voxels).

A comprehensive illustration of the effects in an individual representative subject is provided in Figure 5 by plotting for a single session time courses of upper alpha GFP and BOLD signal from different networks and regions. The HRFconvolved GFP time course in the upper alpha band shows similar fluctuations as the raw BOLD signal time course of the tonic alertness ICN as well as two of its constituent regions. Conversely, the BOLD signal time course of the dorsal attention ICN displays negative correspondence to the alpha GFP fluctuations (Fig. $5 A$ ). The first-level voxelwise mapping of the correlation between BOLD signal and upper alpha GFP delineates the anatomical pattern corresponding to the tonic alertness network (Fig. 5B).

In summary, GFP in the alpha and beta EEG frequency bands showed anatomically selective correlation with fMRI signal fluctuations in the tonic alertness system. Finally, we sought to corroborate our functional interpretation of the cinguloinsular-thalamic network in a dataset acquired in an earlier paradigm setting (Sadaghiani et al., 2009). We used the network defined through positive correlation with alpha power (Fig. 3A) as a spatial mask to extract peristimulus time courses of the previous activation study. We hypothesized that if the system as defined here by EEG correlation underpinned tonic alertness then it should show the same prestimulus effects as previously found for the cingulo-insular-thalamic ICN. Indeed, successful detection of the auditory stimulus compared with misses was preceded by significantly higher prestimulus activity levels in those areas where activity correlates with upper alpha band power (Fig. 6A; at $t=0 \mathrm{~s}$, $t_{(10)}=3.37, p=0.007$, paired 2-tailed $t$ test) and hence closely matched previous results for the related ICN (Fig. $6 B, t_{(10)}=3.86$, $p<0.005)$.

\section{Discussion}

Spontaneous fluctuations of ongoing brain activity can be observed across a wide range of states, from sleep and even sedation, anesthesia, and coma over resting wakefulness all the way to effortful mental activity (Fox and Raichle, 2007). The fluctuations are spatially organized into ICNs. Their spatial patterns remain qualitatively robust across the different states but show finegrained quantitative changes of connectivity (Horovitz et al., 

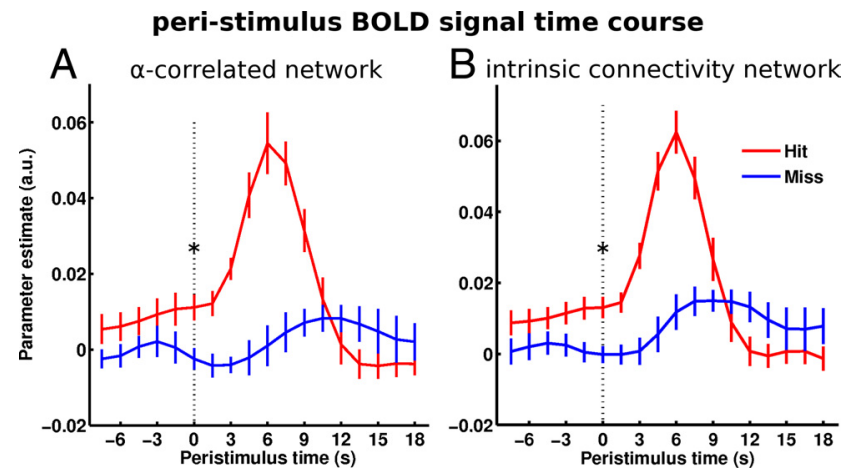

Figure 6. BOLD signal time courses of the tonic alertness system during an auditory detection task. Time courses correspond to successful detection (hit) or miss of repeated identical, near-threshold auditory stimuli (earlier study by Sadaghiani et al., 2009). A, B, Time courses were extracted from the network as defined by positive correlation to upper alpha band power in the current study $(\boldsymbol{A})$ (cf. Fig. $3 A$ ) or by resting state functional connectivity in the subject group of the auditory experiment $(\boldsymbol{B})$. The network defined by positive correlations to alpha shows the same effect as previously observed for the tonic alertness ICN; hits are preceded by significantly higher prestimulus baseline activity compared with miss trials. Note that the spatial pattern of the network as defined by positive alpha correlations was sufficiently robust to be applied as a mask in a different subject group. ${ }^{*} p<0.01$; dashed line indicates stimulus onset.

2009; Vanhaudenhuyse et al., 2010). EEG has a longstanding tradition in monitoring and actually defining different brain states but it has also recently been shown to reflect activity fluctuations occurring within a given state as resting wakefulness. Activity in different ICNs, as recorded for instance in resting state fMRI studies, has been tied to power in different EEG frequency bands or even patterns of power across the EEG spectrum (Mantini et al., 2007). Here, we have pursued a hypothesis derived from previous work and focused on slow activity fluctuations in an as of yet anatomically and functionally insufficiently characterized ICN comprising dACC, anterior insula, thalamus and aPFC (Dosenbach et al., 2007; Seeley et al., 2007). Due to typical coactivation of this network with lateral parietal and prefrontal control regions during paradigm settings, it has stayed elusive whether or not these systems form a unitary or rather two distinct functional networks (cf. Dosenbach et al., 2008 vs Vincent et al., 2008). We show that fluctuations in concurrently recorded electroencephalographic indices of tonic alertness are associated with slow activity fluctuations in the cingulo-insular-thalamic network. This result hence further supports a functional distinction of this network from other control regions.

The most consistent previously reported EEG-hallmark of sustained alertness is the power of alpha oscillations. In a series of experiments, Makeig and colleagues found that lapses in behavioral performance were accompanied by decreases in near $10 \mathrm{~Hz}$ ongoing alpha power (Makeig and Inlow, 1993; Makeig and Jung, 1995). Similarly, Dockree et al. (2007) found sustained levels of near $10 \mathrm{~Hz}$ alpha to reflect tonic maintenance of attentional resources associated with enhancement of phasic goal-directed processing. Likewise, increased alpha phase synchrony (Haig and Gordon, 1998) and power (Gath et al., 1983) have been associated with shorter reaction times in oddball tasks. And sustained increases in upper alpha power correlate with shorter reaction times, less lapses in stimulus detection, and negatively with subjective sleepiness ratings (Lockley et al., 2006). While some of the above findings were obtained in eyes open condition, others were established with closed eyes, indicating that the positive relation between enhanced alpha oscillations and performance is consistent across both conditions. In the present study, we found a positive correlation of GFP in the upper alpha (and to some extent beta) band with activity in the cingulo-insular-thalamic network.

Our findings are overall in accord with results across several previous studies although no single study has so far established the correlation that we show here, namely between alpha band power and an ICN. Positive correlation with alpha band activity has previously been reported for the thalamus (Goldman et al., 2002; Moosmann et al., 2003; de Munck et al., 2007; Ben-Simon et al., 2008; Difrancesco et al., 2008), insula (Goldman et al., 2002) and dACC (Difrancesco et al., 2008). Apart from higher power in our study due to a large study group, differences in the findings may be due to the fact that previous work focused on posterior rather than global alpha and collapsed over lower and upper alpha frequencies.

The correlation of activity in the cingulo-insular-thalamic system with upper alpha band oscillations as the most robust electroencephalographic marker of vigilance fluctuations suggests that this system could serve a role in maintaining tonic alertness. We found similar albeit weaker evidence for beta oscillations that have also been linked to performance in settings requiring sustained alertness (Townsend and Johnson, 1979; Belyavin and Wright, 1987).

As previous related studies discussed above, our analyses were conducted on data recorded during rest where no stimuli or task requirements interfere with the ongoing activity fluctuations that manifest in EEG and fMRI. This advantage for the purpose of our study comes at the price of including no direct measure of perceptual performance in the same dataset. However, the functional interpretation of our findings is supported by previous functional imaging studies that explicitly manipulated tonic alertness and found associated effects in an anatomically very similar network (Sturm and Willmes, 2001; Sturm et al., 2004). Despite qualitative similarity of those latter findings, the present ICN mapping and the network defined by alpha power correlation, this evidence remains indirect. To further validate our conclusions, we hence analyzed fMRI signal time courses in the EEG-defined network but in another subject group previously performing a perceptual experiment requiring tonic alertness (Sadaghiani et al., 2009). Notwithstanding structural intersubject variability between the two study groups, we found that activity in the network linked to upper alpha band power improved perceptual performance with higher prestimulus signal preceding hits compared with misses. This observation further corroborates a close functional correspondence between the cingulo-insular-thalamic ICN, upper alpha band power and tonic alertness.

Tonic alertness refers to a sustained function that is distinct from arousal on the one hand and selective attention on the other hand (Posner, 2008). While arousal (in the sense of wakefulness and responsiveness) is subject to very slow (e.g., circadian) and constitutive modulations and is controlled by the brainstem (Jones, 2008), attention transiently ensures selective local processing of specific features and is tightly linked to activity in dorsolateral parietofrontal cortices (Driver and Frackowiak, 2001). In contradistinction to these cognitive functions, tonic alertness refers to an intermediate capacity that expresses nonselective readiness for perception and action implemented by a corticosubcortical system (Sturm and Willmes, 2001). The anatomy and function of the cingulo-insular-thalamic network is well suited to underpin this control process. The nonspecific thalamic nuclei project very broadly throughout the cortex, qualifying for general functions such as alerting (Scheibel and Scheibel, 1967). Among these, the anterior thalamic nucleus projects massively to the 
dACC, a major cognitive control region implementing performance monitoring and adaptive top-down control (MacDonald et al., 2000; Kerns et al., 2004). Anterior insula with its reciprocal connections to the limbic system and ACC constitutes an anatomical and functional connection hub between extended networks (Sporns et al., 2007; Sterzer and Kleinschmidt, 2010), and has been suggested to dynamically control the switching between internally and externally oriented mental activity (Sridharan et al., 2008).

But apart from the shared correlation with tonic alertness what is the relation between this network and global alpha band power? Note that our findings do not imply the cingulo-insularthalamic network as a generator but rather as a modulator of power in alpha oscillations across the cortex such that this transpires into a GFP signal. Anatomically, thalamo-cortico-thalamic feedback connections are the basis by which the thalamic component of this network might provide an efficient way for controlling global cortical expression of alpha oscillations (Lopes da Silva et al., 1980). Alpha oscillations were the first distinct pattern of ongoing brain activity described in human EEG but their functional significance remains debated. Historically, the Berger effect of alpha disruption by eye opening (Berger, 1929) was taken as indication that this was an "idling" rhythm of the awake but unoccupied resting brain (Pfurtscheller et al., 1996). Apparently in line with this notion, the allocation of selective spatial attention is associated with reductions in alpha activity spatiotopically corresponding to attended locations and enhanced stimulus processing (Rihs et al., 2007). Indeed, we confirmed negative correlations of alpha power with activity in regions related to the control of selective attention, conforming to earlier studies (Laufs et al., 2003).

If interpreted in terms of tonic alertness, our data are nonetheless congruent with an active role for alpha oscillations. We propose that alertness involves a generalized 'windshield wiper' mechanism and that alpha oscillations serve this purpose by rhythmically and synchronously clearing the flood of sensory information on a rapid time scale to reduce distraction and hence enhance detection of novel and relevant sensory information. This proposed mechanism is compatible with evidence of alpha synchronization as an active mechanism for inhibitory top-down control (Klimesch et al., 2007). Enhanced alpha synchronization has been reported in active suppression of task-irrelevant cortical regions in diverse contexts including intermodal selective attention tasks (Foxe et al., 1998), retinotopically specific distractor suppression (Worden et al., 2000; Kelly et al., 2006), featureselective visual working memory tasks (Jokisch and Jensen, 2007), lateralized somatosensory working memory tasks (Haegens et al., 2010) and go/no-go suppression of learned motor sequences (Hummel et al., 2002). In a hierarchical view of attentional function we therefore suggest that both alertness and selective attention serve increased sensitivity but deploy antagonistic mechanisms: Due to its nonselectivity alertness involves what amounts to a suppression and selective attention a specific and focal disruption of this global effect. This view is in accord with the interpretation of alpha desynchronization as a gradual release of inhibition (Klimesch et al., 2007).

The majority of experimental paradigms require the conjunction of alertness and selective attention due to the use of prespecified targets. These settings therefore do not permit to clearly disentangle the contributions from these two attentional functions. For instance, it is not clear whether the expression of alpha oscillations could also be selective and specifically target processes with a high potential for distracting from the task at hand. We propose that a global mechanism is ecologically more useful because not all functional contexts will permit to sharply tune prior assumptions in terms of what to expect as relevant and what as distracting. This question, however, will require future dedicated experiments that orthogonalize the requirements for alertness and selective attention. Some preliminary evidence can be seen in the fact that in our previous study with a nonspatial auditory detection task, there was an antagonistic influence of two usually conjointly task-positive systems on perceptual performance, facilitation by alertness and deterioration by selective spatial attention (Sadaghiani et al., 2009).

\section{References}

Belyavin A, Wright NA (1987) Changes in electrical activity of the brain with vigilance. Electroencephalogr Clin Neurophysiol 66:137-144.

Ben-Simon E, Podlipsky I, Arieli A, Zhdanov A, Hendler T (2008) Never resting brain: simultaneous representation of two alpha related processes in humans. PLoS ONE 3:e3984.

Berger H (1929) Über das Elektroenkephalogramm des Menschen. Arch Psychiatr Nervenkr 87:527-570.

Boly M, Balteau E, Schnakers C, Degueldre C, Moonen G, Luxen A, Phillips C, Peigneux P, Maquet P, Laureys S (2007) Baseline brain activity fluctuations predict somatosensory perception in humans. Proc Natl Acad Sci U S A 104:12187-12192.

de Munck JC, Gonçalves SI, Huijboom L, Kuijer JP, Pouwels PJ, Heethaar RM, Lopes da Silva FH (2007) The hemodynamic response of the alpha rhythm: an EEG/fMRI study. Neuroimage 35:1142-1151.

Difrancesco MW, Holland SK, Szaflarski JP (2008) Simultaneous EEG/ functional magnetic resonance imaging at 4 tesla: correlates of brain activity to spontaneous alpha rhythm during relaxation. J Clin Neurophysiol 25:255-264.

Dockree PM, Kelly SP, Foxe JJ, Reilly RB, Robertson IH (2007) Optimal sustained attention is linked to the spectral content of background EEG activity: greater ongoing tonic alpha $(\sim 10 \mathrm{~Hz})$ power supports successful phasic goal activation. Eur J Neurosci 25:900-907.

Dosenbach NU, Visscher KM, Palmer ED, Miezin FM, Wenger KK, Kang HC, Burgund ED, Grimes AL, Schlaggar BL, Petersen SE (2006) A core system for the implementation of task sets. Neuron 50:799-812.

Dosenbach NU, Fair DA, Miezin FM, Cohen AL, Wenger KK, Dosenbach RA, Fox MD, Snyder AZ, Vincent JL, Raichle ME, Schlaggar BL, Petersen SE (2007) Distinct brain networks for adaptive and stable task control in humans. Proc Natl Acad Sci U S A 104:11073-11078.

Dosenbach NU, Fair DA, Cohen AL, Schlaggar BL, Petersen SE (2008) A dual-networks architecture of top-down control. Trends Cogn Sci 12:99-105.

Driver J, Frackowiak RS (2001) Neurobiological measures of human selective attention. Neuropsychologia 39:1257-1262.

Fox MD, Raichle ME (2007) Spontaneous fluctuations in brain activity observed with functional magnetic resonance imaging. Nat Rev Neurosci 8:700-711.

Fox MD, Corbetta M, Snyder AZ, Vincent JL, Raichle ME (2006) Spontaneous neuronal activity distinguishes human dorsal and ventral attention systems. Proc Natl Acad Sci U S A 103:10046-10051.

Foxe JJ, Simpson GV, Ahlfors SP (1998) Parieto-occipital approximately 10 $\mathrm{Hz}$ activity reflects anticipatory state of visual attention mechanisms. Neuroreport 9:3929-3933.

Gath I, Lehmann D, Bar-On E (1983) Fuzzy clustering of EEG signal and vigilance performance. Int J Neurosci 20:303-312.

Goldman RI, Stern JM, Engel J Jr, Cohen MS (2002) Simultaneous EEG and fMRI of the alpha rhythm. Neuroreport 13:2487-2492.

Gonçalves SI, de Munck JC, Pouwels PJ, Schoonhoven R, Kuijer JP, Maurits NM, Hoogduin JM, Van Someren EJ, Heethaar RM, Lopes da Silva FH (2006) Correlating the alpha rhythm to BOLD using simultaneous EEG/ fMRI: inter-subject variability. Neuroimage 30:203-213.

Haegens S, Osipova D, Oostenveld R, Jensen O (2010) Somatosensory working memory performance in humans depends on both engagement and disengagement of regions in a distributed network. Hum Brain Mapp $31: 26-35$.

Haig AR, Gordon E (1998) Prestimulus EEG alpha phase synchronicity influences N100 amplitude and reaction time. Psychophysiology 35:591-595.

Hesselmann G, Kell CA, Kleinschmidt A (2008a) Ongoing activity fluctua- 
tions in hMT + bias the perception of coherent visual motion. J Neurosci 28:14481-14485.

Hesselmann G, Kell CA, Eger E, Kleinschmidt A (2008b) Spontaneous local variations in ongoing neural activity bias perceptual decisions. Proc Natl Acad Sci U S A 105:10984-10989.

Horovitz SG, Braun AR, Carr WS, Picchioni D, Balkin TJ, Fukunaga M, Duyn JH (2009) Decoupling of the brain's default mode network during deep sleep. Proc Natl Acad Sci U S A 106:11376-11381.

Hummel F, Andres F, Altenmüller E, Dichgans J, Gerloff C (2002) Inhibitory control of acquired motor programmes in the human brain. Brain 125:404-420.

Jokisch D, Jensen O (2007) Modulation of gamma and alpha activity during a working memory task engaging the dorsal or ventral stream. J Neurosci 27:3244-3251.

Jones BE (2008) Modulation of cortical activation and behavioral arousal by cholinergic and orexinergic systems. Ann N Y Acad Sci 1129:26-34.

Kelly SP, Lalor EC, Reilly RB, Foxe JJ (2006) Increases in alpha oscillatory power reflect an active retinotopic mechanism for distracter suppression during sustained visuospatial attention. J Neurophysiol 95:3844-3851.

Kerns JG, Cohen JD, MacDonald AW 3rd, Cho RY, Stenger VA, Carter CS (2004) Anterior cingulate conflict monitoring and adjustments in control. Science 303:1023-1026.

Klimesch W (1999) EEG alpha and theta oscillations reflect cognitive and memory performance: a review and analysis. Brain Res Brain Res Rev 29:169-195.

Klimesch W, Sauseng P, Hanslmayr S (2007) EEG alpha oscillations: the inhibition-timing hypothesis. Brain Res Rev 53:63-88.

Laufs H, Krakow K, Sterzer P, Eger E, Beyerle A, Salek-Haddadi A, Kleinschmidt A (2003) Electroencephalographic signatures of attentional and cognitive default modes in spontaneous brain activity fluctuations at rest. Proc Natl Acad Sci U S A 100:11053-11058.

Laufs H, Holt JL, Elfont R, Krams M, Paul JS, Krakow K, Kleinschmidt A (2006) Where the BOLD signal goes when alpha EEG leaves. Neuroimage 31:1408-1418.

Lockley SW, Evans EE, Scheer FA, Brainard GC, Czeisler CA, Aeschbach D (2006) Short-wavelength sensitivity for the direct effects of light on alertness, vigilance, and the waking electroencephalogram in humans. Sleep 29:161-168.

Lopes da Silva FH, Vos JE, Mooibroek J, Van Rotterdam A (1980) Relative contributions of intracortical and thalamo-cortical processes in the generation of alpha rhythms, revealed by partial coherence analysis. Electroencephalogr Clin Neurophysiol 50:449-456.

MacDonald AW 3rd, Cohen JD, Stenger VA, Carter CS (2000) Dissociating the role of the dorsolateral prefrontal and anterior cingulate cortex in cognitive control. Science 288:1835-1838.

Makeig S, Inlow M (1993) Lapses in alertness: coherence of fluctuations in performance and EEG spectrum. Electroencephalogr Clin Neurophysiol 86:23-35.

Makeig S, Jung TP (1995) Changes in alertness are a principal component of variance in the EEG spectrum. Neuroreport 7:213-216.

Mantini D, Perrucci MG, Del Gratta C, Romani GL, Corbetta M (2007) Electrophysiological signatures of resting state networks in the human brain. Proc Natl Acad Sci U S A 104:13170-13175.

Maris E, Oostenveld R (2007) Nonparametric statistical testing of EEG- and MEG-data. J Neurosci Methods 164:177-190.

Mitra PP, Pesaran B (1999) Analysis of dynamic brain imaging data. Biophys J 76:691-708.
Moosmann M, Ritter P, Krastel I, Brink A, Thees S, Blankenburg F, Taskin B, Obrig H, Villringer A (2003) Correlates of alpha rhythm in functional magnetic resonance imaging and near infrared spectroscopy. Neuroimage 20:145-158.

Pfurtscheller G, Stancák A Jr, Neuper C (1996) Event-related synchronization (ERS) in the alpha band-an electrophysiological correlate of cortical idling: a review. Int J Psychophysiol 24:39-46.

Posner MI (2008) Measuring alertness. Ann N Y Acad Sci 1129:193-199.

Rihs TA, Michel CM, Thut G (2007) Mechanisms of selective inhibition in visual spatial attention are indexed by alpha-band EEG synchronization. Eur J Neurosci 25:603-610.

Sadaghiani S, Hesselmann G, Kleinschmidt A (2009) Distributed and antagonistic contributions of ongoing activity fluctuations to auditory stimulus detection. J Neurosci 29:13410-13417.

Sadaghiani S, Hesselmann G, Friston KJ, Kleinschmidt A (2010) The relation of ongoing brain activity, evoked neural responses, and cognition. Front Sys Neurosci, Advance online publication. Retrieved July 14, 2010. doi:10.3389/fnsys.2010.00020.

Scheeringa R, Bastiaansen MC, Petersson KM, Oostenveld R, Norris DG, Hagoort P (2008) Frontal theta EEG activity correlates negatively with the default mode network in resting state. Int J Psychophysiol 67:242-251.

Scheibel ME, Scheibel AB (1967) Structural organization of nonspecific thalamic nuclei and their projection toward cortex. Brain Res 6:60-94.

Seeley WW, Menon V, Schatzberg AF, Keller J, Glover GH, Kenna H, Reiss AL, Greicius MD (2007) Dissociable intrinsic connectivity networks for salience processing and executive control. J Neurosci 27:2349-2356.

Sporns O, Honey CJ, Kötter R (2007) Identification and classification of hubs in brain networks. PLoS ONE 2:e1049.

Sridharan D, Levitin DJ, Menon V (2008) A critical role for the right frontoinsular cortex in switching between central-executive and default-mode networks. Proc Natl Acad Sci U S A 105:12569-12574.

Sterzer P, Kleinschmidt A (2010) Anterior insula activations in perceptual paradigms: often observed but barely understood. Brain Struct Funct 214:611-622.

Sturm W, Willmes K (2001) On the functional neuroanatomy of intrinsic and phasic alertness. Neuroimage 14:S76-84.

Sturm W, Longoni F, Fimm B, Dietrich T, Weis S, Kemna S, Herzog H, Willmes K (2004) Network for auditory intrinsic alertness: a PET study. Neuropsychologia 42:563-568.

Townsend RE, Johnson LC (1979) Relation of frequency-analyzed EEG to monitoring behavior. Electroencephalogr Clin Neurophysiol 47:272-279.

Vanhaudenhuyse A, Noirhomme Q, Tshibanda LJ, Bruno MA, Boveroux P, Schnakers C, Soddu A, Perlbarg V, Ledoux D, Brichant JF, Moonen G, Maquet P, Greicius MD, Laureys S, Boly M (2010) Default network connectivity reflects the level of consciousness in non-communicative braindamaged patients. Brain 133:161-171.

Vincent JL, Kahn I, Snyder AZ, Raichle ME, Buckner RL (2008) Evidence for a Frontoparietal Control System Revealed by Intrinsic Functional Connectivity. J Neurophysiol 100:3328-3342.

Worden MS, Foxe JJ, Wang N, Simpson GV (2000) Anticipatory biasing of visuospatial attention indexed by retinotopically specific alpha-band electroencephalography increases over occipital cortex. J Neurosci 20:RC63(1-6).

Yuan H, Liu T, Szarkowski R, Rios C, Ashe J, He B (2010) Negative covariation between task-related responses in alpha/beta-band activity and BOLD in human sensorimotor cortex: An EEG and fMRI study of motor imagery and movements. Neuroimage 49:2596-2606. 